\title{
Neurogenic Diabetes Insipidus
}

National Cancer Institute

\section{Source}

National Cancer Institute. Neurogenic Diabetes Insipidus. NCI Thesaurus. Code C84933.

Diabetes insipidus caused by decreased secretion of antidiuretic hormone from the pituitary gland. 